\title{
Experimental setup to study two phase flows for environmental applications
}

\author{
Irina Murgan ${ }^{1}$, Florentina Bunea ${ }^{2, *}$, Adrian Nedelcu ${ }^{2}$, and Gabriel Dan Ciocan ${ }^{1 *}$ \\ ${ }^{1}$ Laboratoire de Machines Hydrauliques, Université de Laval, Loc.1341 Pav. Adrien-Pouliot, Québec, QC, \\ Canada G1V 0A6 \\ ${ }^{2}$ National Institute for R\&D in Electric Engineering ICPE-CA, 313 Splaiul Unirii, 030138, Bucharest, Romania
}

\begin{abstract}
The air water mix is a major concern for the environmental application. This paper proposes an experimental method to accede simultaneously at the water flow velocity field and at the void fraction. Instantaneous and mean fields, as well as the evolution with the flow parameters variation are obtained in cavitation or aerated flows. This method allows a good accuracy for the flow velocity fields $(2 \%)$ and void (vapours or air) contours (4\%).
\end{abstract}

\section{Introduction}

The two-phase flow, and more precisely water - air mix is a key application for the environmental purposes. The water aeration is an important parameter for many environmental application (aeration of water for fish farms, aeration of water passing through hydraulic turbines, water treatment, chemical industry, water cleaning, etc.). For the phenomenological understanding of different phenomena and the characterization of different processes as: dissolved oxygen transfer, mixture of air in water, air transport and transfer, cavitation etc., the validation of transport and transfer models is essential. A particular focus is address in the study of water aeration process - see [1] and bubble column flow [2], [5], [7], where the air bubble morphology is an important parameter for oxygen transfer characterization. The experimental investigation of the two phase flow: water - air is also essential and modern experimental methods like PIV and shadowgraphy are employed to characterize simultaneous both phases, and phases interaction.

This paper presents the application of a PIV method in a close loop test bench. The test bench reproduces the flow at the entrance in a hydraulic turbine draft tube, in cavitational behaviour in fully turbulent flow. In the previous works [3], [7], the contour and velocity of small bubbles were considered. In this paper, a new image processing method is proposed to obtain the vapour pocket contour developed simultaneously with the flow velocity field, with a good accuracy. The instantaneous and mean field are obtained, as well as the evolution of the void fraction with the water flow rate variation.

\section{PIV set-up}

The test bench is a close loop setup that reproduce a rotational, biphasic flow with adverse pressure gradient [3] (Fig.1). This flow reproduces the flow in a draft tube cone of a hydraulic turbine except the rotating velocity component of the runner. The water flow in study area corresponds to Reynolds number in the range $1 \cdot 10^{5} \div$ $5 \cdot 10^{5}$. The divergent effect is obtained using a conic shape with a flaring angle $\gamma=7^{\circ}$, which is correlated with flaring angle of turbine draft tube $\left(\gamma=0^{\circ}\right.$ to $\left.12^{\circ}\right)$ and a stator, placed upstream the conic segment, is use to induce the vortex flow. With the increase of the water velocity, the flow induced by the stator develops a vortex that can become cavitational [4]. An aerator, mounted on the wall of the pipe, downstream the stator, serves to inject dispersed air.

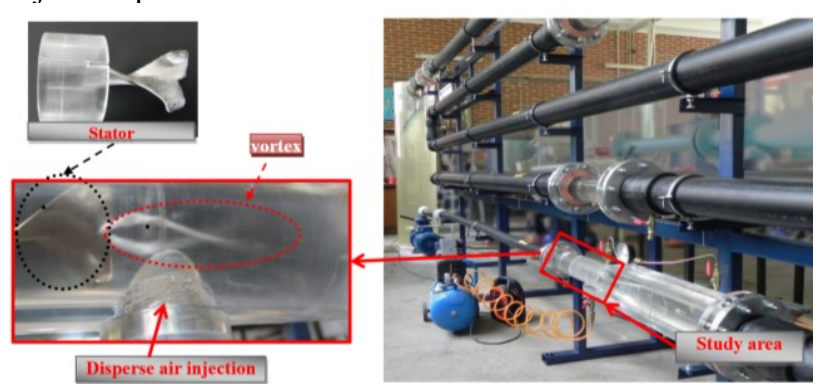

Fig. 1. Test bench for study of rotational biphasic flow with adverse pressure gradient.

The study area, designed for PIV measurements, is made from bulk Plexiglas $\AA$, with plane exterior faces. The interior is machined to simulate conditions in turbine draft tube. Because the Plexiglas ${ }^{\circledR}$ has a refractive index close of the refractive index of the water, 1.49 and respectively, 1.33 for a wavelength of $527 \mathrm{~nm}$. In this way, the optical distortion is minored,

\footnotetext{
*Corresponding authors: buneaflorentina@gmail.com, gabrieldan.ciocan@orange.fr
} 
and it was corrected in post-processing, based on image calibration with a custom target.

The PIV arrangement (Fig. 2) is set up to study both velocity field and vapours volume development (cavitating area) in the symmetry plan of the water passage (2D measurements).

The light source consist on a DualPower TR 15-1000 laser, a pulsed $\mathrm{Nd}$ :YAG laser of $30 \mathrm{~mJ}$ with the wavelength of $527 \mathrm{~nm}$ and pulse duration of $150 \mathrm{~ns}$. The laser source is placed below the study segment with an optical module to create a $3 \mathrm{~mm}$ thick vertical sheet of uniform intensity light through the longitudinal axis of the segment (measurement plane). Laser energy is set to $59 \%$.

The camera, placed perpendicular to the measurement plane, is a FlowSenseEO_4M-32, a monochrome CCD camera with resolution of $2072 \times 2072$ pixels $(7.4 \times 7.4 \mu \mathrm{m}$ pixel pitch) and the bit depth of 12 . The camera is used in double frame mode with exposure time for frame 1 at $15 \mu \mathrm{s}$.

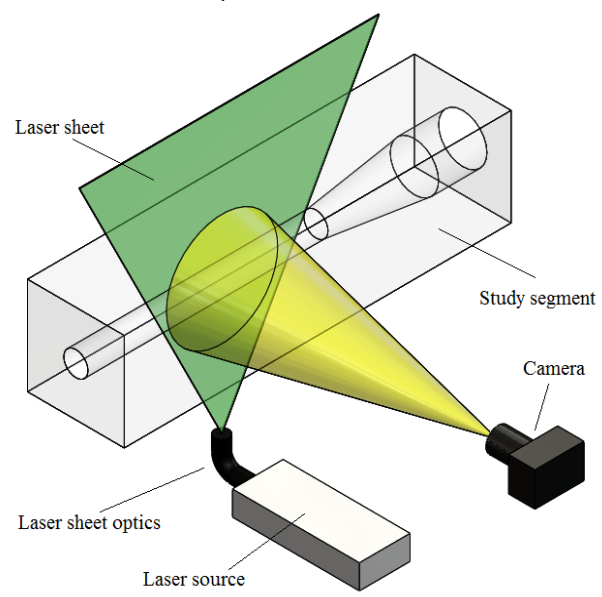

Fig. 2. Schematics of the PIV setup.

The image calibration is customized to fit on the measurement area, with a white dots target (Fig. 3). The rectangular segment of the target, which was in the camera field of view, is $45.83 \times 239 \mathrm{~mm}$ and the dots are $1.5 \mathrm{~mm}$ in diameter and their centres are $5 \mathrm{~mm}$ apart. The target images were processed to obtain the direct linear transformation matrix, which is used to compensate the scale factor variations and the optical distortions between measurement plane and camera sensor. Also, the target was used to align the laser sheet with the measurement plane.

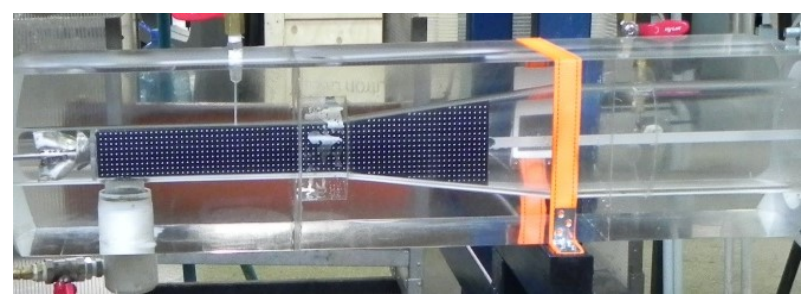

Fig. 3. PIV calibration setup and measure.

As tracers, S-HGS particles - silver coated hollow glass spheres of $10 \mu \mathrm{m}$ in diameter and density of 1.4 $\mathrm{g} / \mathrm{cm}^{3}$, are used. The particles are chosen to respect the flow but also to assure a good SNR (signal to noise ratio) of the image, a clear peak in cross-correlation map (Fig. 4) and a uniform distribution of the tracers.

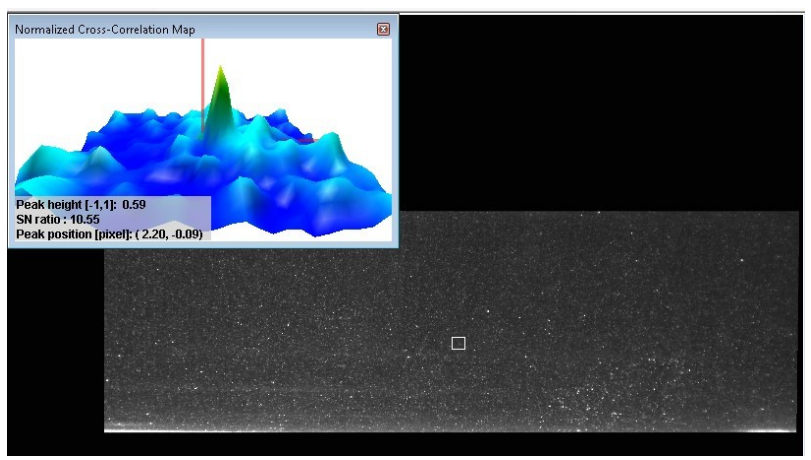

Fig. 4. Image sample and cross-correlation map for the selection.

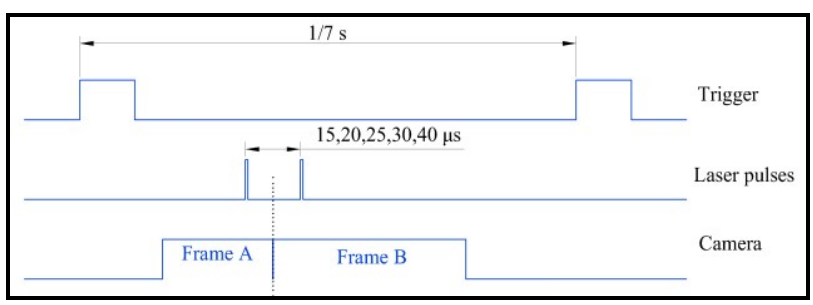

Fig. 5. Timing diagram for synchronization between camera and laser source.

Other components of the PIV system used: BNC 575-8 pulse generator, NI PCIe-1433 camera link, computer with dual CPU Intel(R) Xeon(R) E5-2609 v3 (a) $1.9 \mathrm{GHz}, 32 \mathrm{~GB}$ RAM (16 GB as image buffer) and Dynamic Studio 2015a as software for setup, calibration, acquisition and processing of the image data. The trigger rate for acquisition is $7 \mathrm{~Hz}, 500$ double frame images per run and time between laser pulses is in range $15 \mu \mathrm{s}$ to $40 \mu \mathrm{s}$, adjusted function of the expected mean velocity through study area (Fig. 5).

\section{PIV Data Processing}

\subsection{Velocity Field Processing}

The acquired images are dewarped using the direct linear transformation matrix obtained in calibration stage before applying "Adaptive PIV" analysis module to obtain instantaneous velocity fields in the measurement plane. The velocity results are exported to Matlab for further validation, statistical analysis and representation.

Beside the local validation of the velocity vectors done by DynamicStudio's Adaptive PIV module, using Matlab, a Gaussian filtering was applied on the data series (the 500 velocity fields) to remove the values that do not fit in the bounds:

$$
\bar{u}-3 \sigma \leq \bar{u}_{v a l} \leq \bar{u}+3 \sigma
$$

with $u_{v a l}$ - validated velocity. The standard deviation $(\sigma)$ was calculated as follows for the horizontal and vertical components $u$ and $v$ : 


$$
\sigma_{u}=\sqrt{\frac{1}{n} \sum_{i=1}^{n}\left(u_{i}-\bar{u}\right)^{2}}, \quad \sigma_{v}=\sqrt{\frac{1}{n} \sum_{i=1}^{n}\left(v_{i}-\bar{v}\right)^{2}}
$$

where: $n$ is the number of samples (500 in our case), $u_{i}$, $v_{i}$ - instantaneous velocity components, $\bar{u}, \bar{v}$ - average velocity components.
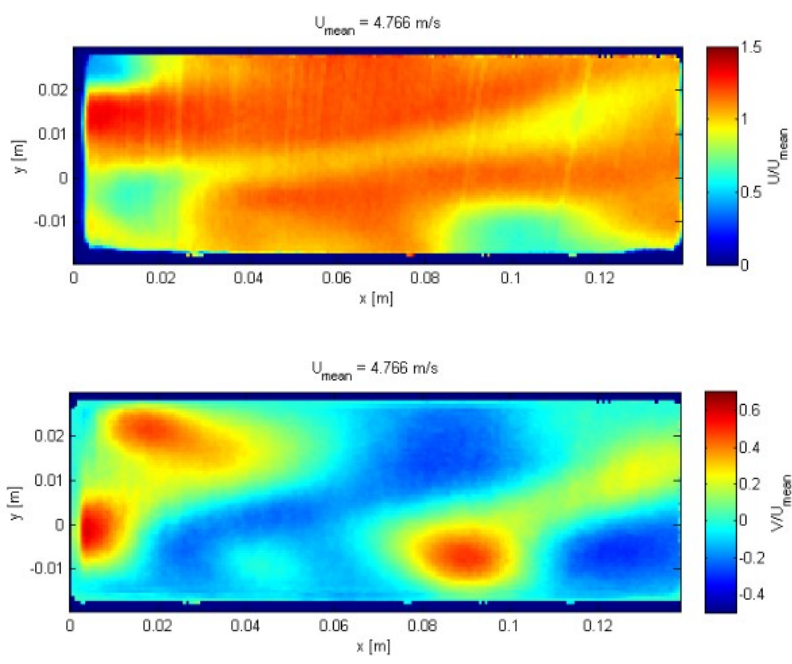

Fig. 6. Water velocity field: axial mean velocity, radial mean velocity.

An example of axial and radial water velocity field is presented in Fig. 6, where $U_{\text {mean }}=4.77 \mathrm{~m} / \mathrm{s}$ is the mean velocity measured with an ultrasound flowmeter. The velocity field obtain by PIV measurement is then given by the mean of validated velocities.

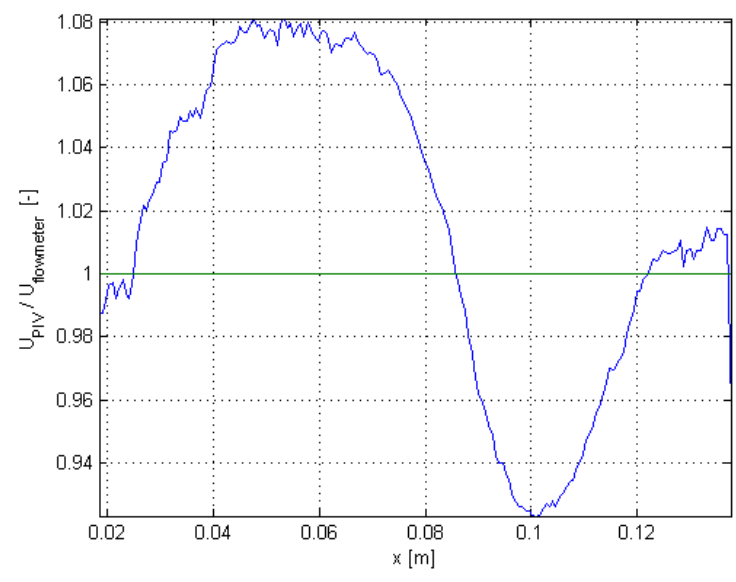

Fig. 7. The PIV mean velocity in different sections, estimated from horizontal component of the velocity, reported to the mean ultrasonic flow measurement.

A global validation (Fig. 7) is done by verifying the flow rate in the section by integrating the horizontal component of the velocities:

$$
Q=\int_{y} u_{x} d A
$$

where $u_{x}$ - velocities at coordinate $x, d A$ - area element in which $u_{x}$ is measured. The integrated velocity profile was compared with an ultrasonic flow rate measurement in one phase flow. As shown in [8], the swirl velocity field is not axial symmetric, and the velocity nonuniformity due to the vortex in the measurement plane is $8 \%$ of the mean field. The global accuracy of the PIV velocity is better that $2 \%$.

\subsection{Image Processing}

Specific techniques of image processing are used in order to identify the percentage of vapours in each measurement condition.

The procedure of air slug flow detection is similar to the image processing bubble detection used in [5]. Hence, for the present PIV data, the principal steps of the detection approach (Fig. 8) are the following: the improvement of the images quality by diminishing the effect of the PIV particles, the separation of image's interest areas by setting a binarization threshold, and the air percentage estimation.

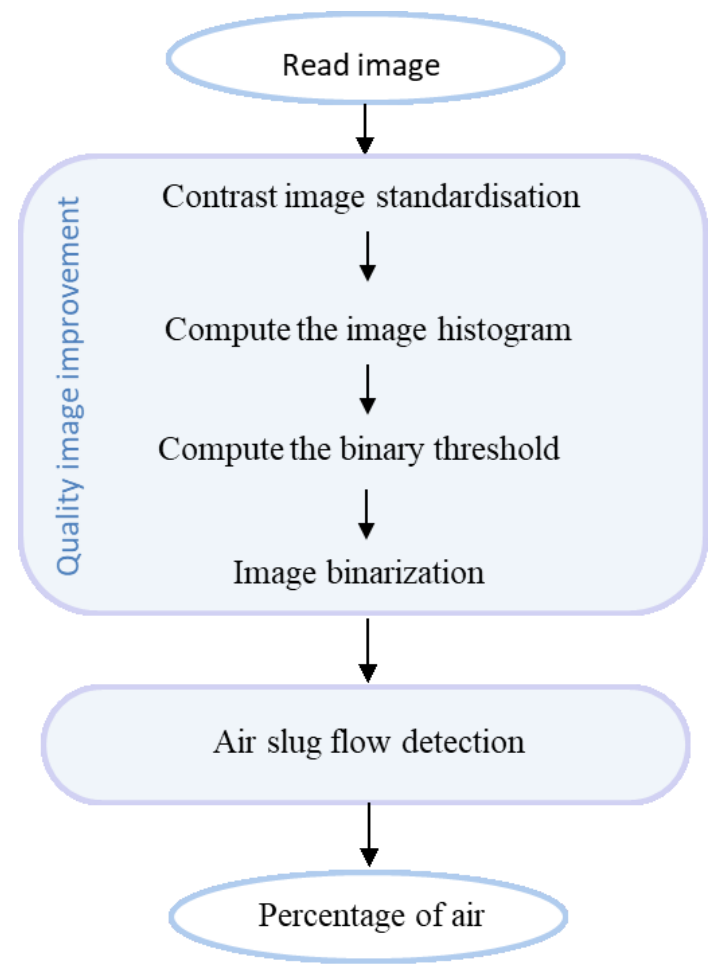

Fig. 8. The image processing procedure for percentage of vapours estimation, implemented in Matlab software.

\subsubsection{Quality image improvement}

In the context of the slug flow detection, the grayscale images acquired using the PIV system are considered to be polluted by the presence of the particles (Fig 9.a.), contrary to their purpose. This is why, the first step is to reduce as much as possible the effect of the particles on the air slug detection, by replacing the brightened pixels with very low intensity grey level, taking into consideration the surrounding pixels grey level. After image contrast adjustment [6], the image Gaussian shaped histogram (Fig. 9.b) indicates the presence of an object well separated from the image background. 


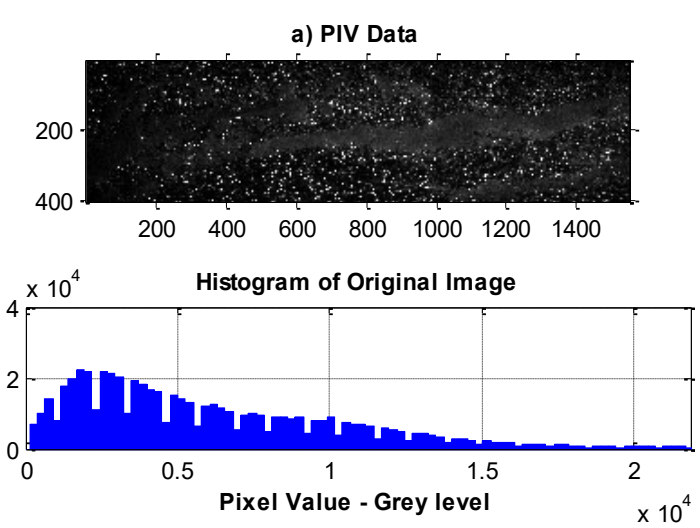

b) Quality improved image
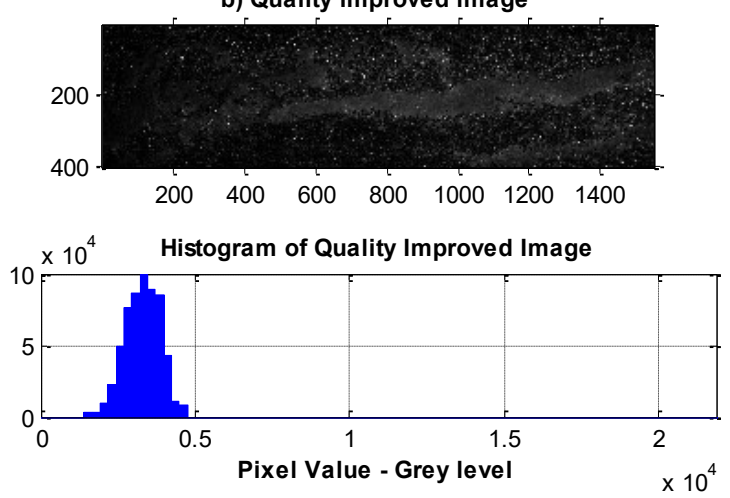

Fig. 9. Original PIV data-image (a) and the quality improved one (b), with their corresponding pixels histograms.

\subsubsection{Image binarization}

The object indicated by the grayscale image histogram corresponds to the vapor slug on which detection we are interested. For each quality improved image, a threshold is applied in order to select the zones of interest (Fig. 10). The binarization threshold is actually an interval given by the histogram mean $\pm 5 \%$. In the case of PIV data acquired with no air injection, a global threshold is used, compared with the one acquired with air injection where the adaptive thresholding is preferred. In this second case, the supplementary air injection influences the images non-uniform lightening and a global threshold will not give proper results on the same image.
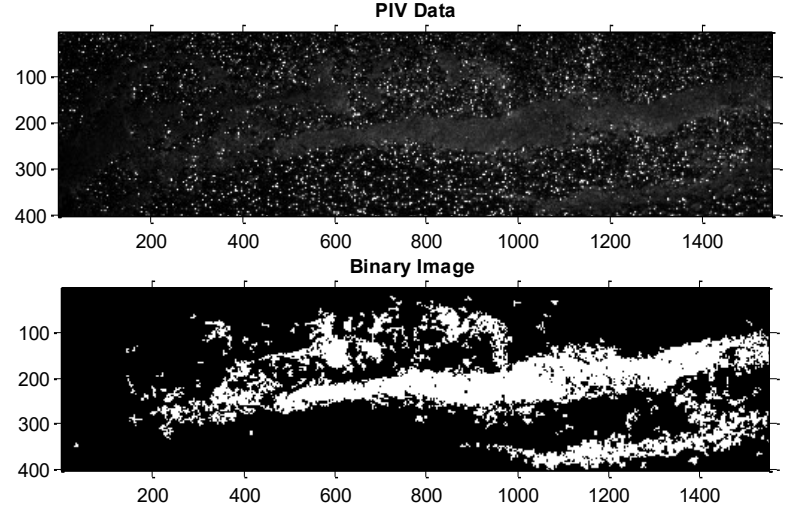

Fig. 10. Binary image using global threshold for PIV measurements without air injection.

\subsubsection{Vapours fraction estimation}

The white pixels from the binary image shown in figure 10, represent the air slug flow. Computing the percentage of white pixels with respect to the size of the flow domain (measured also in pixels), the flow air fraction is obtained.

A mean position of the cavitational vapours pocket can be represented for each flow configuration. Finally, the mean vapour pocket contour can be superposed on the mean velocity field corresponding of a flow configuration as shown in Fig 11.
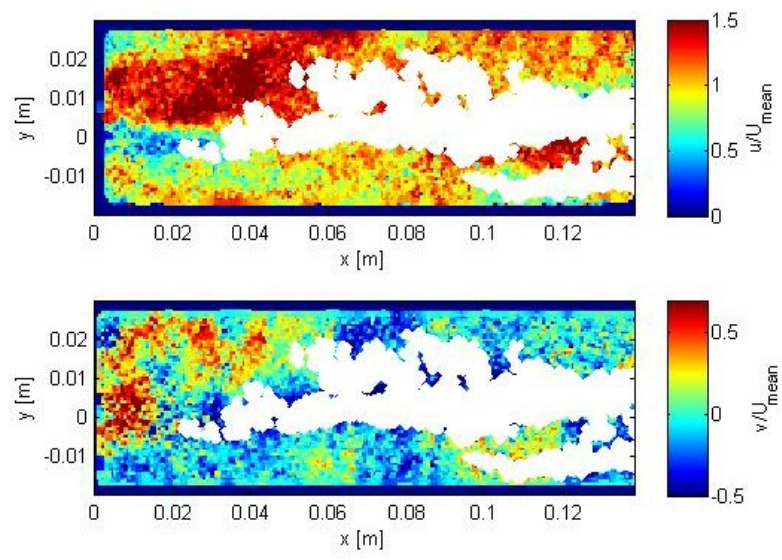

Fig. 11. Instantaneous axial and radial mean flow velocity field and the corresponding cavitational vapours pocket.

In this way the evolution of the vapour fraction can be correlated with the cavitation development in the section - see fig 12 .

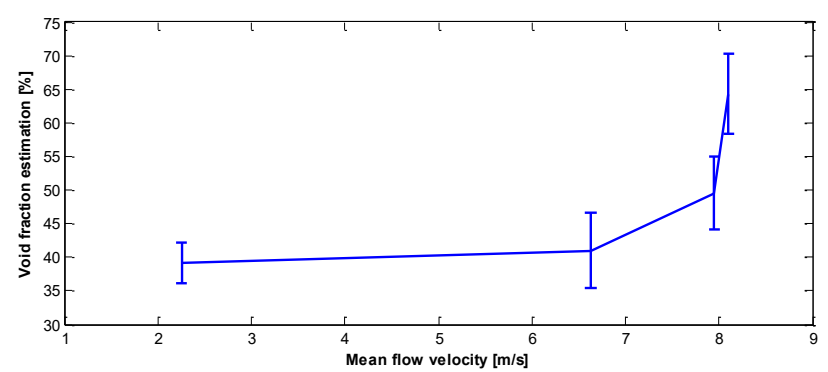

Fig. 12. Cavitation development variation (void fraction) with the increase of the flow velocity, without air injection.

\section{Conclusions}

An experimental method to measure the void fraction, simultaneous with the water velocity filed was developed.

By PIV data processing, the instantaneous and mean flow velocity field and the corresponding void fraction are estimated in rotating turbulent flows, for different regimes: cavitating or with aeration. The results shown a good accuracy, as well as for the velocity field (2\%) and void fraction (4\%). The evolution of the cavitation regimes or aeration parameters was correlated with the flow parameters.

Future works will be performed for the implementation of the $3 \mathrm{D}$ velocity field measurements. 
The work has been funded by the Executive Unit for Financing Higher Education, Research, Development and Innovation (UEFISCDI), PN-III-P1-1.2-PCCDI-2017， ctr. 81/2018, TEACHERS project.

\section{References}

1. F. Bunea, G. Ciocan, Experimental study of standard aeration efficiency in a bubble column, Desalination and Water Treatment Murat Eyvaz and Ebubekir Yüksel, IntechOpen, 349-368 (2018) DOI: 10.5772/intechopen.76696

2. G. Besagni, F. Inzol, T. Ziegenhein Two-Phase Bubble Columns: A Comprehensive Review, ChemEngineering (2018), 2, 13; doi: $10.3390 / 2020013$

3. F. Bunea, A. Nedelcu, G.D. Ciocan, Prediction of water aeration efficiency in high turbulent flow, Desalination and Water Treatment, 85, 55-62, (2017),

4. A. Digulescu, I. Murgan, F. Bunea, G.D. Ciocan, D.M. Bucur, G. Dunca, I. Candel, C. Ioana, Cavitating vortex characterization based on acoustic signal detection, IOP Conf. Series: Earth and Environmental Science, 49 (2016) 082009

5. I. Murgan, F. Bunea, G. Ciocan, Experimental PIV and LIF characterization of a bubble column flow, Flow Measurement and Instrumentation 54, 224235m (2017)

6. L. Tan, J. Jiang, Digital Signal Processing Fundamental and Applications, $2^{\text {nd }}$ ed., Elsevier Publishing Academic Press, (2013)

7. A.M. Aliyu, H. Seo, H. Kim, K.C. Kim, Characteristics of bubble-induced liquid flows in a rectangular tank, Experimental Thermal and Fluid Science, 97, 21-35, (2018)

8. M.S. Iliescu, G.D. Ciocan, F. Avellan, Analysis of the cavitating draft tube vortex in a Francis turbine using particle image velocimetry measurements in two-phase flow, Journal of Fluids EngineeringTransactions of the ASME,130 2, (2008). 